\title{
La cobertura regional del movimiento estudiantil chileno 2011: prensa impresa y prensa digital en La Región de Los Ríos (Chile)
}

\author{
Rodrigo BROWNE SARTORI \\ Universidad Austral de Chile \\ rodrigobrowne@uach.cl \\ Pamela Romero LizAma \\ Universidad Alberto Hurtado (Chile) \\ pamelarome@gmail.com \\ Sofía Monsalve GuARDA \\ Universidad Austral de Chile \\ sofia.monsalveg@gmail.com
}

Recibido: 24 de septiembre de 2014

Aceptado: 24 de marzo de 2015

\begin{abstract}
Resumen
La presente investigación pretende descubrir, mediante el Análisis Crítico del Discurso (ACD), los procesos y formas de representación de los actores sociales vinculados al movimiento estudiantil chileno en el año 2011, a través del análisis de la cobertura regional de "El Diario Austral Región de Los Ríos" y el diario electrónico "El Naveghable", durante junio, octubre y diciembre de 2011. La labor de esta investigación está orientada a describir cómo los medios de comunicación locales de la ciudad de Valdivia construyeron realidad, diálogo, opinión, actores sociales, prejuicios y estereotipos en la cobertura del movimiento estudiantil regional.
\end{abstract}

Palabras clave: prensa escrita, prensa digital, movimiento estudiantil, Chile.

\section{Regional coverage of the Chilean student movement 2011: Print Newspapers and online media in Los Ríos Region (Chile)}

\begin{abstract}
This research aims to discover, through critical discourse analysis (CDA), the processes and forms of representation of social actors linked to the Chilean student movement in 2011, through the analysis of regional coverage of "El Diario Austral Región de Los Rios" and the electronic journal "El Naveghable" during June, October and December 2011. The work of this research is aimed at describing how local media in the city of Valdivia constructed reality, dialogue, review, social actors, prejudices and stereotypes in covering the regional student movement.

Keywords: newspapers, digital press, student movement, Chile.

\section{Referencia normalizada}

BROWNE SARTORI, Rodrigo; ROMERO LIZAMA, Pamela; y MONSALVE GURADA, Sofía (2015): "La cobertura regional del movimiento estudiantil chileno 2011: prensa impresa y prensa digital en La Región de Los Ríos (Chile)”. Estudios sobre el Mensaje Periodístico. Vol. 21, Núm. 2 (julio-diciembre), págs.: 723-740. Madrid, Servicio de Publicaciones de la Universidad Complutense.
\end{abstract}

Sumario: 1. Introducción. 2. Discusión teórica; 2.1. Medios de comunicación; 2.2. Educación en el Chile actual; 2.3. Movimientos estudiantiles en Chile y el gran movimiento del 2011; 2.4. Medios de comunicación y nuevos movimientos juveniles. 3. Metodología; 3.1. Fundamentación; 3.2. Ejemplo de aplicación metodológica. 4. Resultados generales; 4.1. Diario El Austral Región de Los Ríos; 4.1.1. Junio 2011; 4.1.2. Octubre 2011; 4.1.3. Diciembre 2011; 4.2. Diario Digital El Naveghable; 4.2.1. Junio 2011; 4.2.2. Octubre 2011; 4.2.3. Diciembre 2011. 5. Conclusiones. 6. Referencias bibliográficas. 


\section{Introducción}

Miquel Rodrigo Alsina (2005: 62) señala que los medios de comunicación son un lugar de constante confrontación entre diversos actores, ya sean productores de la comunicación, fuerzas políticas o grupos económicos internos y externos, los cuales intervienen tanto en la producción y circulación de la información. Pueden ser fuentes de diálogo, ser fieles a la concepción de agentes de responsabilidad social con la comunidad y buscar mejoras para ésta o promover violencia, desorden, manipulación y centralización de la información.

Los medios de comunicación constituyen un espacio en el que se decide y se ejerce una influencia en la percepción, la construcción de la realidad y de los hechos noticiosos en los receptores del medio a través de la selección de la información, el uso de las fuentes, el uso de la imagen y la interpretación realizada por el propio periodista.

En este sentido, los medios pueden dirigir efectivamente la percepción de la realidad cuando no se dispone de una señal contrainformativa. Y, aunque los medios no puedan moldear cada opinión, sí pueden enmarcar la realidad perceptiva en torno a la cual se forman las opiniones. Aquí radica tal vez su efecto más importante: establecer el orden del día para todos, organizando el espacio de lo público, las cuestiones en qué pensar. En suma, los medios establecen los límites del discurso y de la comprensión del público. No siempre moldean la opinión de todos, claro está, pero tampoco tienen por qué hacerlo (Romano, 1991: 36-37).

La industria mediática imperante en Chile está centralizada, por lo que la mayoría de las informaciones se concentran en Santiago, debido a que los grandes medios de comunicación (televisión, periódicos y radios) se encuentran en la capital del país.

Hoy en día se está viviendo un cambio de paradigma, puesto que los diferentes medios incluyen en su discurso a los actores sociales que no tienen injerencia en las altas esferas político-económicas, invitándolos a ser un testigo o un caza noticia, aunque la estructura de la información o hechos noticiosos ya está establecida, como es tradicional, por la agenda setting del medio.

A pesar de ello, aún se entrega una visión que interesa al medio, como sucedió en el año 2011 con la cobertura del movimiento estudiantil chileno, enfocándose en hechos violentos o de farándula, en vez de las razones importantes detrás de la movilización.

En este caso, la industria mediática chilena no sólo contribuyó a la generación de opiniones o de construcciones mentales sobre el movimiento estudiantil, sino que acalló las voces de los actores sociales en cuestión, especialmente la de los estudiantes. Los jóvenes que fueron parte del conflicto en el año 2011 fueron estereotipados por los medios, por presiones políticas o ideologías de los mismos agentes de información.

Los medios de comunicación social tuvieron un rol fundamental en la difusión de los principales hechos que enmarcaron este fenómeno. Esta información fue tema obligado en noticieros de televisión, radio y de diferentes programas, además de titulares de diversos diarios nacionales y locales, cuando el problema dejó de estar centralizado en la capital del país.

Es aquí donde la industria mediática jugó un papel fundamental en el desarrollo e información del movimiento estudiantil, tomando una posición cercana a actores so- 
ciales o identidades dominantes, dejando u obviando una parte de la realidad, y destacando diversas fuentes, como autoridades, personal policial, académicos y rectores de las universidades y escuelas.

En este ámbito, esta investigación pretende determinar cómo dos medios locales, específicamente El Diario Austral Región de Los Ríos y el diario electrónico El Naveghable, cubrieron y trataron el Movimiento Estudiantil Chileno en el año 2011, a nivel local. Para ello se utilizará como herramienta metodológica el Análisis Crítico del Discurso (ACD), con el cual se observa el tratamiento periodístico de la información en la producción de noticias sobre la movilización estudiantil en Valdivia, durante los meses de junio, octubre y diciembre de 2011.

\section{Discusión teórica}

\subsection{Medios de comunicación}

Los medios de comunicación son constructores de realidad mediante la entrega de información al público, la que permite entender el mundo y crear opinión sobre hechos noticiosos. Son considerados como instituciones legitimadas y con alto grado de credibilidad, producen significados que se graban poderosa e indeleblemente en la conciencia de las personas y se constituyen como conocimiento que se reafirmará en todo el entramado simbólico de la cultura. Actúan sobre un mundo referencial ya existente en los individuos, el cual puede ser modificado mediante la realidad creada por éstos mismos.

Internet ha ampliado las funciones de los medios de comunicación de masas. Los diarios de hoy en día son condicionados en la morfología del mensaje y en el uso de éste, el lector ya no está limitado a las ediciones periódicas impresas sino que podrá revisarlas de manera on line cuando le apetezca e inclusive puede generar su propia información y realidad del hecho.

Este avance ha generado un mayor empoderamiento de los medios de comunicación como agentes transmisores de noticias y de realidades, ya que son ellos quienes imponen la agenda informativa. Incluso si el medio no dice nada sobre un tema o acontecimiento, este no existirá en el temario personal o en el espacio cotidiano. Los medios ejercen un tipo de biopoder en los seres humanos, que comienza con la misma apropiación del tiempo (Romano, 2006: 10).

Los medios de comunicación no sólo han sentido las incursiones tecnológicas sino que también la influencia de la economía capitalista, convirtiéndolos en empresas que están insertas en un sistema de economía de mercado que tiende a unificar su práctica productiva. Por tanto, los medios buscan crear un público que aporte tiempo, dinero y se inserte en la masificación de los recursos tecnológicos para recibir la gran cantidad de mensajes y disponer de ellos a favor de los propietarios de las nuevas tecnologías.

Bechelloni (1986: 381) señala que los medios del siglo XXI están estructurados en su funcionamiento mediante tres lógicas: La primera de "cultura de masas", una estandarización y repetición de contenidos, generando modas y consumo. La segunda corresponde al "periodismo de actualidad", el cual se articula a través de los géneros de noticia y temas de actualidad, basándose en la credibilidad. Rodrigo Alsina (2005: 
223) se refiere a esta segunda lógica, donde los medios establecen un contrato pragmático fiduciario con la sociedad, en el cual se asume que la información entregada por estos es verídica, invitando a la audiencia a confiar en el discurso informativo que publican.

La tercera lógica corresponde a la "del servicio", donde los medios de comunicación han olvidado sus funciones sociales al servicio público, contribuyendo al funcionamiento de los sistemas democráticos. No obstante ésta queda en el olvido debido a que están insertos en el contexto económico productivo capitalista. La industria mediática persigue tres tipos de objetivos: El primero incrementar la audiencia. Segundo incrementar la publicidad (estos se han convertido en difusores de anuncios publicitarios algunos relacionados con la información y otros con el entrenamiento). El tercer objetivo no está relacionado con el beneficio, como por ejemplo, objetivos culturales o educativos.

La construcción de los medios de comunicación sobre qué hay que pensar, lo realizan a través de una selección de hechos interpretando y construyendo de manera subjetiva una comunicación mediada por la realidad interpersonal del periodista al momento de elaborar una noticia. Es ahí donde el comunicador transmite información, crea significados e imágenes en los receptores. "Los medios de comunicación no son un mediador neutral, lógico o racional de los acontecimientos sociales o de la realidad, sino que ayudan básicamente a reproducir ideologías reformuladas" (Connell, 1980, citado en van Dijk, 1990: 28).

\subsection{Educación en el Chile actual}

El sistema educacional en Chile se originó durante la dictadura militar de 1973, en el cual se promulgó la Ley Orgánica Constitucional de Enseñanza (LOCE), donde el Estado desempeño un rol regulador y delegó parte de la educación al sector privado. Los puntos que proponía la LOCE eran que la educación es un derecho de todas las personas, la que deben cumplir y ejecutar preferentemente los padres de familia respecto de sus hijos; otorgando al Estado el deber de prestar especial protección al ejercicio de este derecho; y, en general, a la comunidad, el deber de contribuir al desarrollo y perfeccionamiento de la educación.

Esta ley, significó que los docentes perdieran el estatus de empleados públicos. En materia de financiamiento, y fiel a la ideología predominantemente neoliberal del régimen militar, se instauró el nuevo sistema de subvención por alumno (voucher), o "subvención a la demanda", y se incentivó el ingreso de proveedores particulares que competían por esta subvención fiscal.

Se confiaba en que las fuerzas de mercado (la competencia entre los proveedores por los alumnos y la libre entrada y salida de establecimientos) harían lo suyo para resolver tanto el problema de cobertura como el de calidad. El objetivo era entregar mayor libertad a los consumidores (padres y apoderados), descentralizar la administración y aumentar la eficiencia y calidad en el uso de recursos.

La descentralización significó que las municipalidades se encargaban de administrar el personal docente e instalaciones y el Ministerio velaba por el currículum y los aspectos pedagógicos. El objetivo fue incentivar la descentralización y hacer que las 
escuelas respondieran a las necesidades locales. Ninguno de estos objetivos logró cumplirse, pues sólo se descentralizaron algunas funciones administrativas.

Con el regreso de la democracia, se siguió manteniendo este sistema educacional, pero en el año 1996 se llevó a cabo una nueva reforma curricular y comenzó la jornada escolar completa, luego de complejos acuerdos entre gobierno y oposición. Sin embargo este sistema seguía generando una inequidad y desigualdad en las oportunidades de la educación.

Los diversos parches que se han realizado a la educación chilena no han posibilitado un cambio profundo de ésta. Por ende, en el año 2006, los estudiantes secundarios realizaron un paro de sus actividades con motivo de mejorar la calidad de la educación en el país y que el Estado se haga cargo de ella. Debido a esa gran movilización, la presidenta en ese entonces, Michelle Bachelet, perteneciente al Partido Socialista, promulgó una nueva que ley que reemplazaba a la Ley Orgánica Constitucional de Enseñanza creada durante la Dictadura Militar.

Ahora pasaba a existir la Ley General de la Educación, la cual definía a la educación como el proceso de aprendizaje que abarca las distintas etapas de la vida de las personas y que tiene como finalidad alcanzar su desarrollo ético, moral, solidario, afectivo e intelectual, mediante la transmisión de valores, conocimientos. Esta nueva ley enunciaba los objetivos de la educación chilena: Universalidad y educación permanente, Calidad de la Educación, Equidad del Sistema Educativo, Participación, Responsabilidad, Articulación del sistema educativo, Transparencia y Flexibilidad.

Además propició los marcos normativos que regulan la educación en el país actualmente: a) Una institucionalidad pública más sólida que permita dotar a nuestra Educación de un sistema de aseguramiento de la calidad; b) Definición clara de los roles de cada uno de los actores de la comunidad educativa; c) Una nueva estructura curricular para elevar la calidad de la educación; d) Garantizar la eficiencia y la transparencia de todo el sistema educacional; e) Asegurar y desarrollar la autonomía; f) Normas de no discriminación arbitraria y selección; g) Incremento en la subvención escolar, tanto rural como urbano, h) Un nuevo Consejo Nacional de Educación; i) Mantener y desarrollar un sistema educacional de provisión mixta.

A pesar de estos cambios en la educación chilena, la situación no ha tenido mejoras relevantes y sus actores sociales han vuelto a manifestar este descontento.

En el año 2011, los estudiantes secundarios y universitarios comenzaron una movilización a nivel nacional, desde el mes de junio (el movimiento surgió en abril pero las movilizaciones masivas entre los dos entes juveniles comenzaron poco después) de aquel año hasta fines de noviembre. Los secundarios solicitaban una mejor calidad en la educación, cambios en la estructura en lo público y los universitarios solicitaban educación gratuita y de calidad, además del fin del lucro de las universidades.

La magnitud de este movimiento era desconocida en la historia del país y de antiguos movimientos estudiantiles realizados en distintos años de existencia de Chile como una república democrática, que incluso dieron paso a movilizaciones internacionales como en Colombia, donde los estudiantes manifestaban por una educación universitaria gratuita la cual les fue otorgada.

La crisis educacional en Chile no ha tenido una solución. El gobierno de Sebastián Piñera no dio respuestas contundentes a los estudiantes, en tanto que en el 2014, ya 
con Michelle Bachelet nuevamente como presidenta, el gobierno presentó al poder legislativo para su discusión proyectos de ley para una reforma educacional, que busca entregar educación de calidad para los niños, niñas y jóvenes de Chile, pero que no ha obtenido el apoyo de los representantes de movimientos sociales y estudiantiles, ni de los sectores políticos del país vinculados a la derecha.

Entre los cambios propuestos se presentan los siguientes: a) Fin al lucro y fortalecimiento del rol fiscalizador del Estado; b) Fin al copago, o financiamiento compartido; c) Fin a la selección escolar; d) Creación de la Subsecretaría de Educación Parvularia y la Intendencia de Educación Parvularia; e) Creación del administrador provisional y administrador de cierre de instituciones de educación superior y regulaciones en materia de administración provisional de sostenedores educacionales.

\subsection{Movimientos estudiantiles en Chile y el gran movimiento del 2011}

En la historia de las movilizaciones estudiantiles en el país, éstas se han caracterizado por ser protagonizadas por estudiantes de la educación superior, identificados con problemas gremiales y políticos.

La primera movilización estudiantil se suscitó en junio del año 1931, en la Casa Central de la Universidad de Chile por parte de estudiantes que protestaban en contra del presidente Carlos Ibáñez del Campo. Aquella movilización estuvo dirigida por la Federación de Estudiantes de la Universidad de Chile y la Federación de Estudiantes de la Universidad Católica de Chile.

Luego, en la década del 60, se presenciaron diversas movilizaciones de estudiantes universitarios, las cuales estuvieron marcadas por el ingreso a la educación superior de jóvenes provenientes de clase media baja y obrera. Durante ese periodo se suscitó la reforma universitaria, que era considerada por los estudiantes como un proceso de transformación profunda de la universidad, con el fin de aprovechar la ciencia, la educación superior y la cultura como instancias de cambio social para construir una sociedad más justa y solidaria.

Otra manifestación ocurrió en el año 1987, durante la Dictadura Militar, donde los alumnos de la Universidad de Chile se movilizaron debido a la designación del rector José Luis Federici, hecho que manifestó el repudio de los estudiantes, llevando a la renuncia de la máxima autoridad de la casa de estudios.

Posteriormente, se vuelve a suscitar otra movilización estudiantil en el año 2006 bajo el Gobierno de la presidenta Michelle Bachelet, con un carácter diferente a las anteriores, integrándose los secundarios. Este gran movimiento fue conocido como "la Revolución Pingüina" (se conoce popularmente en Chile a los estudiantes de establecimientos municipalizados como "pingüinos" por sus uniformes). Los secundarios buscaban reivindicaciones puntuales como la gratuidad de la Prueba de Selección Universitaria (PSU), pase escolar gratuito en el transporte para la educación media, y un cuestionamiento profundo a la reforma educativa chilena y a los fundamentos mismos del sistema escolar en el país.

Los líderes del movimiento pingüino llamaron a un paro nacional, el cual fue acogido por todos los estudiantes secundarios del país. Se estima que para el 30 de mayo del año 2006 había 250 colegios paralizados sólo en Santiago. Tras esta jornada el 1 
de junio, la presidenta Michelle Bachelet anunció un paquete de medidas destinadas a mejorar la calidad de la educación, como la reforma a la LOCE, la formación de un consejo asesor presidencial para la educación, pase escolar gratuito, PSU gratis para el $80 \%$ más pobre y becas. No obstante, lo propuesto por el ejecutivo no fue bien recibido por los jóvenes y anunciaron un nuevo paro nacional el 5 de junio de 2006.

Sin embargo, este movimiento no progresó debido a que mostró una descoordinación en la asamblea estudiantil y entre los dirigentes, además de la estrategia utilizada por el Gobierno, solicitando que los medios de comunicación no cubrieran en demasía la movilización. El 9 de junio de 2006 los estudiantes secundarios anunciaron el fin de las movilizaciones a nivel nacional.

Se preveía que después de esta "revolución pingüina" no existiría otra movilización estudiantil en Chile, pero ocurriría todo lo contrario y surgiría un movimiento que unificará a los estudiantes secundarios y universitarios, quedando en la historia de Chile como uno de los movimientos más importantes y relevantes.

El conflicto se comenzó a suscitar durante los meses de abril y mayo de 2011 en algunas universidades de Santiago. El primer hecho ocurrió en la Universidad Central de Chile (UCEN) donde los estudiantes paralizaron sus actividades debido a la modificación en los estatutos de la universidad, ya que dejaría de ser una corporación sin fines de lucro.

A su vez, el otro hecho que movilizó a los universitarios fue el caso de la Universidad Tecnológica Metropolitana (UTEM), donde el programa de la Televisión Nacional de Chile (TVN) "Esto no tiene nombre" mostró un reportaje que denunciaba supuestas irregularidades en la casa de estudios superiores. Este acontecimiento suscitó manifestaciones en contra de la universidad y del Ministro de Educación del momento, Joaquín Lavín.

La primera gran movilización se llevó a cabo, entonces, el 1 de junio de 2011 mediante la Confederación de Estudiantes de Chile (CONFECH). La marcha indicaba que este movimiento sería distinto de los anteriores, no solo por la unificación de secundarios y universitarios sino que además por la participación de rectores de universidades como Juan Manuel Zolezzi (rector USACH) y Luis Pinto (rector UTEM), además de la Agrupación Nacional de Empleados Fiscales (ANEF).

Este movimiento demostraba una organización y unión jamás vista en otras movilizaciones estudiantiles a nivel nacional. La Confech dejó en libertad de decisión a las federaciones de las otras universidades del país para adherirse al paro. Sin embargo, al 3 de junio había alrededor de 17 universidades que se encontraban en paro de sus funciones como la Universidad de Playa Ancha (Valparaíso), Universidad de La Serena, Universidad Arturo Prat (Iquique), Universidad Católica de Valparaíso, Universidad de Magallanes (Punta Arenas), Universidad de Valparaíso, Universidad del Bío Bío (Concepción), Universidad de Concepción, Universidad Católica de la Santísima Concepción, Universidad de Santiago de Chile, Universidad de Atacama (Arica), Universidad Católica del Norte (Antofagasta), Universidad de La Frontera (Temuco), Universidad de Los Lagos (Osorno), Universidad Federico Santa María (Valparaíso) y la Universidad de Chile.

A su vez, los secundarios también se unían de manera masiva a las movilizaciones. Al 26 de junio había 600 colegios en paro. Ante este crecimiento, el Ministro Lavín 
solicitó deponer las tomas de todos los establecimientos educacionales e iniciar un diálogo con las autoridades.

Durante los primeros meses del movimiento, el Gobierno del presidente Sebastián Piñera comunicó reformas educacionales para satisfacer las demandas estudiantiles, estás medidas se centraron en el proyecto GANE (Gran Acuerdo Nacional de la Educación) y el FE (Fondo por la Educación). Las medidas ofrecidas por las autoridades de Gobierno no enfocaban el problema principal que solicitaban los estudiantes. Se seguía manteniendo el mismo sistema de educación y la influencia del neoliberalismo en ella. Las autoridades políticas no querían reconocer la crisis que vivía la educación chilena.

"El no asumir que estamos ante un sistema socioeducativo altamente segmentado e inequitativo evidencia la poca pertinencia de estas medidas para la realidad de nuestro país, lo que hace dudar de la intención por mejorar la calidad de todos los establecimientos, sobre todo los municipales. Las evaluaciones estandarizadas no aseguran calidad y propiciarán una competencia aún más descarnada por obtener buenos puntajes para recibir mayor financiamiento. La semaforización de los colegios promoverá la concentración de los mejores estudiantes y conllevará a una mayor exclusión, elitización y reducción del sistema público" (Vallejo, 2012: 37).

No obstante, la posición de los estudiantes se endureció aún más siguiendo con las movilizaciones a nivel nacional y así comenzaron a suscitarse otros hechos que caracterizaron el movimiento, como la aparición de los jóvenes líderes universitarios Camila Vallejo, Giorgio Jackson y Camilo Ballesteros. También las marchas de los días jueves realizadas en todo el país y el carácter de que en cada región se seguía el petitorio nacional pero se incluían en los diálogos con las autoridades regionales, además de las necesidades locales.

El movimiento no sólo derribó las costumbres habituales de difusión por los medios de comunicación, ya que éstos fueron ignorados por los estudiantes, siendo éstos mismos los que generaron sus propios medios de información sobre sus propias movilizaciones, por internet y redes sociales. A su vez, los medios de comunicación generaron un discurso sensacionalista sobre el movimiento, enfocándose en las marchas y destacando los incidentes entre estudiantes, encapuchados anónimos y personal policial. "Los medios de comunicación que pretenden calumniar nuestras movilizaciones, se han visto desprestigiados y han sido reemplazados por las redes sociales como fuentes de información verídica y oportuna" (Vallejo, 2012: 62).

A pesar de esto, las universidades a nivel nacional se seguían adhiriendo al paro, como la Universidad Austral de Chile, en Valdivia. Para mantener vivo el movimiento los estudiantes realizaron diversas manifestaciones lúdicas para incorporar a la sociedad en el tema educacional.

En la Región de Los Ríos se evidenció el mismo problema denunciado por los dirigentes estudiantiles a nivel nacional, sobre la cobertura de los medios de comunicación que cayeron en un sensacionalismo que exacerbaba hechos violentos, destrozos, enfrentamientos con la policía e incluso atribuyéndoles estereotipos como terroristas, al ser denunciados por el uso de elementos para la construcción de bombas caseras y artesanales. 


\subsection{Medios de comunicación y nuevos movimientos juveniles}

Para hablar de los movimientos sociales, primero debemos comprender qué son. Existen diversas definiciones para este concepto. Según Giddens (en Feixa, 2002: 10) los movimientos sociales son un intento colectivo de promover un interés común o de asegurarse un objetivo compartido, mediante la acción en masa en el exterior de la esfera de las instituciones establecidas.

Otra definición la propone Eric Neveu (2000), en la cual hace referencia a los movimientos sociales como un concepto difícil de precisar, a la vez que cuestiona algunos de sus supuestos elementos tales como el político, ya que plantea que cada uno tiene sus propias características. Por ende es complejo dar una definición exacta sobre los movimientos sociales ya que cada uno de ellos tiene sus propias características que lo hacen único y diferente.

Los jóvenes siempre han estado involucrados en diversos procesos sociales como actores secundarios o protagonistas. Su participación ha sido constante, mediante movimientos netamente estudiantiles, movilizaciones en apoyo a la clase obrera, protesta en contra de un Gobierno o simplemente por modos de vida como la generación de subculturas conocidas como tribus urbanas, anarquistas, metaleros, veganos, punk, entre otros.

Los movimientos de épocas anteriores eran muy distintos a las movilizaciones de la nueva cultura juvenil, han cambiado las bases de estos y las han adaptado a formas desconocidas para la sociedad, las cuales han generado una revolución jamás antes vista después del famoso mayo del 68.

Rosana Reguillo (2003) hace referencia a estas nuevas culturas juveniles modernas, con una capacidad de asociación y de actuar socialmente distinta a lo conocido, con percepciones políticas actualizadas, tanto del espacio como de lo público. La autora menciona las características de estos nuevos movimientos sociales realizados por la cultura juvenil moderna: a) Poseen una conciencia globalizada; b) Priorizan los pequeños espacios de la vida cotidiana como campos de lucha para impulsar una transformación global; c) Se respeta la heterogeneidad; d) Selección cuidadosa de las causas sociales en las que se involucran; e) El barrio o el territorio han dejado de ser epicentro del mundo.

Según Neveu (2000), los nuevos movimientos sociales generados por esta nueva cultura juvenil moderna rompen con el funcionamiento de las estructuras sindicales y de partido, desafían a los fenómenos centralizadores que delegan a la autoridad a lejanos "Estados Mayores" en beneficio de la asamblea general y del control de los dirigentes.

Debido a estas nuevas características de las culturas juveniles modernas, la sociedad no ha logrado comprender los nuevos movimientos sociales surgidos en el siglo XXI, han abandonado las estructuras de las antiguas movilizaciones y han desarrollado nuevas formas de lucha y de comunicación. Como consecuencia, los diversos actores de la sociedad no han sabido como relacionarse y enfrentar a estas solicitudes reclamadas por los jóvenes.

Esto ha quedado demostrado con la "revolución pingüina" de 2006, en la cual los estudiantes secundarios manifestaron sus peticiones a las autoridades sobre un cambio en la educación chilena, pase escolar gratuito y cambios en la PSU. El desarrollo de este 
movimiento tenía la característica de las nuevas movilizaciones, una descentralización, una autonomía y una resistencia hacia los gobernantes como al control social.

Los líderes no sólo representaban a los estudiantes sino que manifestaban abiertamente sus preceptos políticos, el joven actual no sólo es un alumno sino que además pasa a integrar otros ámbitos de la sociedad, como es el de la política.

Neveu (2000) señala que los movimientos sociales expresan los malestares sociales, las modificaciones culturales y revelan el surgimiento de solidaridades colectivas o la dispersión de los grupos cuya coherencia acabó por parecer evidente; por eso, son a la vez una constante de la vida social y un fenómeno que cambia sin cesar.

Los nuevos movimientos sociales vienen con una carga adquirida en el ámbito político, ya que principalmente siempre tienen que ver con esta tendencia, es decir, se hace un llamado a las autoridades políticas a que respondan ante los requerimientos planteados por estos nuevos actores e inclusive se imputa a las autoridades la responsabilidad de los problemas que originaron la movilización. No obstante cabe recordar que los movimientos no pueden considerarse netamente políticos.

En el caso de Chile, se ha visto una proliferación de los nuevos movimientos sociales que han ido cambiando en su estructura y en la forma de organización y desarrollo de las movilizaciones. El año 2011 se suscitó el conflicto estudiantil más relevante en la historia del país, este concentró a los estudiantes secundarios y universitarios con una sola bandera de lucha, una educación de calidad en el ámbito secundario y gratuito en lo referente a lo universitario.

El conflicto tenía una estructura que lo hacía diferente al ocurrido en el año 2006, el nivel de organización, de liderazgo y descentralización llevo a éste a alcanzar ribetes inesperados para las autoridades, los integrantes de la sociedad y los medios de comunicación.

Este movimiento derribó las concepciones de los antiguos movimientos estudiantiles en el país, siendo un fenómeno totalmente desconocido para las autoridades como para los demás integrantes de la sociedad. La movilización del 2011 fue descentralizada, autónoma, proponiendo nuevas medidas, con una resistencia al control social y además con una exclusión a los medios de comunicación tradicionales.

Camila Vallejo (2012), la principal líder y figura de este movimiento, indica que los jóvenes decidieron asumir un rol importante que involucra a toda la sociedad sobre la educación que se imparte en el país y el rol de las universidades, a la cual la sociedad chilena ha cerrado sus ojos y en la cual ha hecho crítica a los gobiernos del país que no han solucionado la crisis que vive el sistema educacional chileno.

Para Camila Vallejo, la universidad es un agente vivo en su construcción y en el desarrollo del proyecto de país que los ciudadanos movilizados tratan de levantar día a día, haciendo énfasis en la responsabilidad de generar organización al interior de ella, para así transformar la universidad y la sociedad.

\section{Metodología}

\subsection{Fundamentación}

La noticia no puede ser considerada como un reflejo fiel de la realidad ya que ésta es una construcción del periodista. En ella se transfiere el punto de vista y la interpreta- 
ción de los hechos por parte del comunicador, además de la ideología del medio de comunicación que la produce. Teun van Dijk (1990: 9) señala "que las noticias deben estudiarse principalmente como una forma de discurso público". En su posición, los discursos son fundamentales a la hora de la expresión y reproducción de ideologías en el diario vivir. Lo importante es ver cómo las ideologías marcan y determinar los distintos niveles y estructuras discursivas.

El Análisis Crítico del Discurso (ACD) se presenta como una alternativa para conocer de qué manera se expresa la ideología por medio de los textos. En esta línea, la presente investigación pretende descubrir y evidenciar el tratamiento informativo que se realizó durante el movimiento estudiantil 2011 en la Región de Los Ríos en la cobertura del diario de prensa escrita El Austral de Los Ríos y el diario electrónico El Naveghable. Para ello se utilizó en una primera etapa metodológica una matriz planteada en el proyecto Fondecyt $11070062^{1}$ y el proyecto de Pluralismo en el Sistema Informativo chileno PLU $11010^{2}$, en base a los estudios elaborados por Teun van Dijk (1990 y 1999). La matriz mencionada se construye de la siguiente manera:

\begin{tabular}{|c|c|c|}
\hline \multicolumn{2}{|c|}{ 1. Plano significativo/texto } & 2. Plano formal/texto/contexto \\
\hline \multicolumn{2}{|c|}{ 1.1. Nivel Temático: significados globales } & 2.1. Estructuras formales sutiles \\
\hline \multirow{2}{*}{$\begin{array}{l}\text { 1.2. Nivel de significados } \\
\text { locales }\end{array}$} & a) de carácter implícito o indirecto & \multirow{2}{*}{ 2.2. Nivel Contextual } \\
\hline & b) de carácter explícito o directo & \\
\hline
\end{tabular}

Los significados globales permiten evidenciar cuáles son los temas a los que se le da importancia para así poder obtener una idea general de la noticia analizada. En los significados locales se busca el significado literal de las palabras mediante construcciones discursivas en la noticia, que posteriormente crean modelos mentales y opiniones en base a las construcciones sociales realizadas por quienes construyen la información.

Este ámbito se divide en dos: a) Carácter implícito o indirecto: Informaciones que se pueden inferir de palabras del texto como implicaciones, presupuestos, alusiones, ambigüedades, hipérboles; b) Carácter explícito o directo: Informaciones evidentes que se encuentran con claridad en las palabras del texto. Se busca indagar acerca de las estrategias léxicas utilizadas por los periodistas.

1 Comunicación intercultural y periodismo intercultural: análisis crítico de la construcción social de la realidad a través de la representación mapuche y peruano-boliviana en las noticias de la prensa diaria de cobertura nacional (Copesa y El Mercurio) - 2008. Comisión Nacional de Investigación Científica y Tecnológica (CONICYT). Ministerio de Educación de Chile.

2 El movimiento estudiantil 2011: Diversidad temática y complejidad discursiva en la construcción de nuevas actorías. Estudio comparado de la prensa regional impresa y digital de Valparaíso, La Araucanía y Los Ríos - 2012. Fondo de Estudios sobre Pluralismo en el Sistema Informativo. Comisión Nacional de Investigación Científica y Tecnológica (CONICYT). Ministerio de Educación de Chile. 
El Plano Formal Texto-Contexto está divido en dos planos, el primero es Estructuras Formales Sutiles y el Nivel Contextual. A través de la primera se pretende observar cómo opera lo ideológico en el discurso, por lo cual se pueden emitir falacias, omitir información y construir discursos dominantes que no son percibidos debido a su sutileza pero que influyen en la interpretación y creación de la propia realidad del otro.

Por su parte, en el nivel contextual se busca observar las representaciones mentales generadas en el discurso noticioso que influyen en la memoria a largo plazo de la audiencia, en la cual se almacenan conocimientos y opiniones sobre las experiencias propias.

Para la segunda etapa metodológica de esta investigación, se realiza una comparación entre los discursos periodísticos generados por El Diario Austral Región de Los Ríos y el diario electrónico El Naveghable, además de ver las diferencias y semejanzas en la información noticiosa entregada por los diarios regionales. Es aquí donde se podrá evidenciar las influencias, la generación de tópicos y las representaciones mentales que producen las noticias sobre los diferentes actores sociales del movimiento estudiantil 2011, en los discursos mediáticos generados por los periodistas.

Para el análisis de las noticias se seleccionaron tres meses (junio, octubre y diciembre) que evidencian diferentes periodos del movimiento estudiantil en Los Ríos y distintos discursos mediáticos realizados por ambos medios locales, que demuestran diversas realidades de un mismo hecho y que con el transcurrir de los meses cae en un sensacionalismo que estereotipa a los estudiantes.

En junio se comenzó con las grandes movilizaciones, toma de los establecimientos educacionales, ya sea secundarios como universitarios, difusión de demandas, y se mostraba una gran cohesión a nivel nacional como regional. Luego en octubre se comienza a evidenciar un debilitamiento del movimiento, los estudiantes universitarios y secundarios empiezan a tomar rumbos diferentes, como el plebiscito de la Universidad Austral de Chile (UACh), movilizaciones con poca participación y grupo antitoma en la UACh. Posteriormente, en diciembre, la cobertura periodística comienza a decaer en ambos diarios y el movimiento estudiantil ya enfrenta un nuevo proceso, como es el regreso a clases de los estudiantes y las marchas esporádicas.

\subsection{Ejemplo de aplicación metodológica}

Para comprender mejor la herramienta metodológica, los resultados y las conclusiones de esta investigación, se expondrá un ejemplo que explica cómo se aplicó la matriz antes expuesta.

\begin{tabular}{|l|l|l|}
\hline \multicolumn{1}{|c|}{$\begin{array}{c}\text { Fecha: } \\
\text { 02 de Junio de 2011 }\end{array}$} & \multicolumn{2}{c|}{ EI Naveghable } \\
\hline \multicolumn{3}{|c|}{ TITULARES } \\
\hline Título & $\begin{array}{l}\text { Más de medio millar de estudiantes } \\
\text { marcharon pidiendo por mejoras en la } \\
\text { educación pública. }\end{array}$ & $\begin{array}{l}\text { Estudiantes marcharon por } \\
\text { Valdivia y se reunieron en la } \\
\text { Seremi de Educación }\end{array}$ \\
\hline Bajada & No tiene & $\begin{array}{l}\text { Más de 500 estudiantes } \\
\text { universitarios y secundarios se } \\
\text { adhirieron a la marcha nacional } \\
\text { convocada por la Confech, en } \\
\text { demanda por la democratización } \\
\text { universitaria y el fin al lucro. }\end{array}$ \\
\hline
\end{tabular}




\begin{tabular}{|c|c|c|c|}
\hline Periodista & \multicolumn{2}{|c|}{ Luis Contreras León } & Marcos Labra \\
\hline Fuentes & \multicolumn{2}{|c|}{$\begin{array}{l}\text { Presidente de la Feuach, Rodrigo Salazar } \\
\text { Secretaria general de la Feuach, Cari Álvarez } \\
\text { Vicepresidente de la Feuach, Rodrigo Cosimo } \\
\text { Presidenta Feesva, Jean Maureen Fuentes } \\
\text { Seremi de Educación, Carlos Crot } \\
\text { Jefa de Gabinete Seremi de Educación, } \\
\text { Claudia Bottner }\end{array}$} & $\begin{array}{l}\text { Presidente de la Feuach, Rodrigo } \\
\text { Salazar }\end{array}$ \\
\hline \multicolumn{4}{|c|}{ ANÁLÍSIS COMPARATIVO } \\
\hline \multirow{3}{*}{ 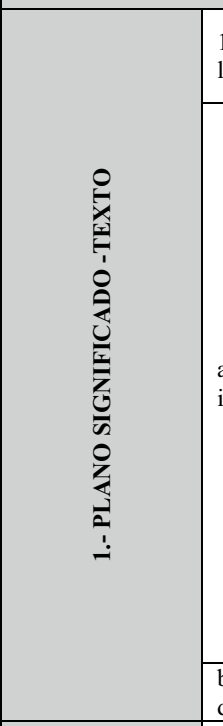 } & $\begin{array}{l}\text { 1.2. Nivel de significados } \\
\text { locales: }\end{array}$ & \multirow[b]{2}{*}{$\begin{array}{l}\text { Más de medio millar de } \\
\text { estudiantes marcharon } \\
\text { pidiendo mejoras en la } \\
\text { educación pública. } \\
\text { Se reconoce una gran } \\
\text { participación y adhesión de } \\
\text { los estudiantes al conflicto } \\
\text { estudiantil en Los Rios. } \\
\text { En la misiva, los estudiantes } \\
\text { universitarios critican la } \\
\text { actuación del ministro de } \\
\text { Educación Joaquín Lavín al } \\
\text { plantear una serie de } \\
\text { reformas a la educación } \\
\text { superior sin tomar en cuenta } \\
\text { las opiniones de los actores } \\
\text { sociales. } \\
\text { Se muestra a los estudiantes } \\
\text { como críticos o inquisidores } \\
\text { ante el accionar de las } \\
\text { autoridades de gobierno al } \\
\text { no tomar en cuenta a los } \\
\text { estudiantes. }\end{array}$} & \multirow[b]{2}{*}{$\begin{array}{l}\text { Estudiantes marcharon por } \\
\text { Valdivia. } \\
\text { Se indica que los estudiantes } \\
\text { se movilizaron representando } \\
\text { a la ciudad pero no al } \\
\text { movimiento estudiantil, } \\
\text { obviando que los estudiantes } \\
\text { se manifestaron por la } \\
\text { Educación, por una } \\
\text { democratización } \\
\text { universitaria y por el fin al } \\
\text { lucro. } \\
\text { Como exitosa consideraron } \\
\text { los dirigentes estudiantiles la } \\
\text { marcha realizada esta tarde } \\
\text { en Valdivia. } \\
\text { Se enfatiza que sólo desde el } \\
\text { punto de vista de los } \\
\text { estudiantes la marcha tuvo } \\
\text { gran participación, donde se } \\
\text { demuestra que el periodista } \\
\text { de manera irónica se refiere } \\
\text { a la jornada. }\end{array}$} \\
\hline & $\begin{array}{l}\text { a) De carácter implícito o } \\
\text { indirecto }\end{array}$ & & \\
\hline & $\begin{array}{l}\text { b) De carácter explícito o } \\
\text { directo }\end{array}$ & No hay resultados & No hay resultados \\
\hline $\begin{array}{l}0 \\
0 \\
0\end{array}$ & $\begin{array}{l}\text { 2.1.- Estructuras formales } \\
\text { sutiles }\end{array}$ & $\begin{array}{l}\text { Se habla de un movimiento } \\
\text { estudiantil masivo pero la } \\
\text { imagen que se construye de } \\
\text { los estudiantes es de críticos, } \\
\text { de justicieros ante las } \\
\text { autoridades de gobierno, al } \\
\text { pedir explicaciones del } \\
\text { actuar de estos. }\end{array}$ & $\begin{array}{l}\text { Se minimiza la movilización } \\
\text { estudiantil y además se da a } \\
\text { conocer la información como } \\
\text { si los estudiantes estuvieran } \\
\text { manifestándose por Valdivia, } \\
\text { como si fuera una } \\
\text { competición, obviando que } \\
\text { los jóvenes entregaron un } \\
\text { petitorio con demandas a las } \\
\text { autoridades regionales. }\end{array}$ \\
\hline $\begin{array}{l}\text { 3.- RESULTADOS: } \\
\text { TÓPICOS DE } \\
\text { TEXTO }\end{array}$ & \multicolumn{3}{|c|}{$\begin{array}{l}\text { El Diario Austral de Los Ríos informa la movilización de los estudiantes } \\
\text { universitarios y secundarios de la Región como masiva, con adhesión, no obstante } \\
\text { se presenta a los estudiantes exigiendo explicaciones a las autoridades. } \\
\text { En tanto, El Naveghable, entrega la misma información pero desde otro punto de } \\
\text { vista, minimiza el movimiento entregando una cifra de } 500 \text { personas protestando y } \\
\text { enfocándose a que los jóvenes marcharon por Valdivia y no por la educación. }\end{array}$} \\
\hline
\end{tabular}

\section{Resultados generales}

\subsection{Diario El Austral Región de Los Ríos}

De las noticias sobre el movimiento estudiantil de 2011 en el Diario El Austral de Los Ríos durante junio, octubre y diciembre, se analizaron 88 textos informativos. El mes de junio cuenta con 41 noticias, periodo de apogeo máximo del movimiento en los medios de comunicación. Octubre fue el mes de transición del conflicto estudiantil y en él se estudiaron 36 noticias. Por último, en el mes de diciembre se analizó una muestra de 11 noticias que relatan los momentos de finalización del movimiento. 
El Austral de Los Ríos, es el único periódico impreso y de tiraje diario en Los Ríos, este fue fundado el 18 de noviembre 1982 y pertenece a la Sociedad Periodística Araucanía (S.A.P.) de la cadena de diarios regionales de El Mercurio. Este medio funciona principalmente con periodistas titulados lo que otorga más veracidad al tratamiento informativo de las noticias debido a la formación y ética de los profesionales de la comunicación.

\subsubsection{Junio 2011}

La totalidad de las noticias publicadas en junio construyeron diversos discursos influenciados por estereotipos hacia los estudiantes y utilizando palabras que califican el actuar de los jóvenes como violento o encapuchados, calificaciones que permiten la creación de tópicos en los lectores sobre los principales actores sociales de este movimiento. No obstante, esta situación cambia cuando se refieren a las fuentes de autoridades políticas y de educación, el discurso es más benevolente.

En relación a los tópicos de texto, se destacan los siguientes, relacionados a dos actorías sociales:

a) Estudiantes: Críticos; Inquisidores; Autoritarios; Amenazantes; Delincuentes; Rebeldes; Indolentes, Borrachos; Destructores; Criminales; Violadores.

b) Autoridades de Gobierno: Victimas; Benevolentes; Dialogadores; Preocupados; Garantes.

\subsubsection{Octubre 2011}

El discurso generado en el mes de octubre tiene una similitud con el presentado en junio, a partir de las últimas semanas donde se suscitaron los hechos más significativos del movimiento. Los tópicos destacados fueron los siguientes:

a) Estudiantes: Flojos; Exigentes; Rebeldes; Delincuentes; Encapuchados; Violentos; Discriminadores; Cúpula de Poder (referente a la Confech).

b) Autoridades: Pocos serios; Falta de presencia; Cómplices; Garantes del actuar de Carabineros.

c) Carabineros: Garantes del orden público; Castigadores; Exceso de Poder.

\subsubsection{Diciembre 2011}

En el presente mes el movimiento estudiantil ha venido disminuyendo, los estudiantes han retomado sus actividades lectivas y con marchas esporádicas, tanto a nivel nacional como regional. Los discursos son varios con diversas interpretaciones de los distintos actores sociales. A continuación:

a) Estudiantes: Autoritarios; Secuestradores del bien público; Lucradores; Intransigentes; No dialogantes.

b) Autoridades: Dialogantes; Garantes; Víctimas; Preocupadas.

\subsection{Diario Digital El Naveghable}

El Naveghable es el primer diario electrónico de Los Ríos que trabaja mediante la participación ciudadana en la creación de su información. Este medio es propiedad de Inversiones y Publicaciones Comunidades Ciudadanas Sociedad Anónima y miembro 
de la red de diarios ciudadanos Mi Voz. Las noticias que son creadas por la comunidad son revisadas por el editor periodista Marcos Labra, además de las publicaciones realizadas por la periodista Fernanda Duque. A su vez, cuentan con la opción de corresponsales, es decir, ciudadanos reporteros sin ninguna formación periodística.

El total de noticias a analizar durante los meses de junio (6), octubre (5) y diciembre (3) de 2011, es de 14. A continuación se detallan los resultados obtenidos en el análisis crítico del discurso de este medio de comunicación ciudadano.

\subsubsection{Junio 2011}

Durante el inicio del movimiento estudiantil en Valdivia, los discursos propuestos por el diario son diversos y otros parecidos a los del diario El Austral de Los Ríos. No obstante, demuestran otra interpretación de la realidad, con otras miradas de un mismo hecho.

a) Estudiantes: Autoritarios; Sin Razón, Sin Diálogo.

b) Autoridades: Dialogantes; Honorables; Preocupadas.

\subsubsection{Octubre 2011}

En el presente mes se repite la misma situación y se vuelven a construir diversos discursos abarcando variadas temáticas que representan una realidad, aunque ésta tenga un carácter sensacionalista. A continuación se detallan los discursos obtenidos de los análisis de las noticias pertenecientes al mes en cuestión.

a) Estudiantes: Incitadores; Autoritarios; Irrespetuosos; Provocadores.

\subsubsection{Diciembre 2011}

En este mes las noticias han decaído, los estudiantes han retomado sus actividades lectivas y los discursos se reducen -pero con una gran diferencia hacia el otro medio tradicionalista- porque se reconocen hechos en contra de los estudiantes y, por tanto, cambiando la imagen de las autoridades.

a) Estudiantes; Violentos; Destructores; Dialogantes; Democráticos.

b) Autoridades: Mentirosos, Castigadores; Represores.

\section{Conclusiones}

En base a la pregunta realizada en esta investigación sobre cómo fue el tratamiento informativo que dieron El Diario Austral Región de Los Ríos y el diario electrónico El Naveghable al movimiento estudiantil en la Región de Los Ríos (Chile), podemos concluir que hubo un discurso social imperante sobre el movimiento estudiantil y sus diferentes actores sociales por parte de El Diario Austral, debido, principalmente, a la mayor lectoría en la ciudad y la cantidad de noticias analizadas, y a que por su parte El Naveghable es un medio electrónico consultado más por generaciones jóvenes que por la mayoría de los habitantes de la comunidad valdiviana.

Debido a esta situación, el discurso construido durante los meses junio, octubre y diciembre fue predominante para el periódico impreso El Diario Austral, por lo que la mayoría de las representaciones mentales y opiniones estuvieron marcadas por esta visión única de la realidad. 
En lo referente al tratamiento informativo de El Diario Austral está investigación arrojó que el medio tomó la misma postura informativa que los medios nacionales, "estereotipar" al movimiento estudiantil, generando un discurso que pasó por diferentes procesos: informativo, sensacionalista, encapuchados y debilitamiento del conflicto estudiantil.

Burguet (2004) señala que los medios de comunicación no son intermediarios neutrales, puesto que siempre optan por una postura, ya sea por la línea editorial o por la posición que se tome por parte de actores influyentes, por ende, se destaca que estos no son notarios de la realidad, sino que meros intérpretes.

La interpretación de la realidad del movimiento estudiantil en El Diario Austral estuvo marcada por tres grandes discursos informativos: movilizaciones, tomas de establecimientos educacionales por secundarios, minimización de la movilización universitaria, criminalización del movimiento y en menor cantidad a aquellos temas relacionados con gestión, declaraciones o demandas.

En tanto, en El Naveghable su construcción discursiva era contradictoria, ya que en las noticias elaboradas por los periodistas se caía en la criminalización de los estudiantes y en destacar hechos de desorden o enfrentamientos con Carabineros. No obstante, existía una contrainformación por parte de los corresponsales ciudadanos quienes informaban los hechos de manera distinta o desmentían lo que El Diario Austral Región de Los Ríos publicaba.

Durante el inicio de las movilizaciones estudiantiles en la Región, ambos diarios comenzaron sus discursos de manera informativa sobre las principales demandas y características del conflicto estudiantil. Se fueron presentando los diversos actores sociales que eran partícipes de la movilización de los estudiantes.

Se mostró un movimiento articulado, descentralizado, masivo, con liderazgo, reticente al control social, democrático e inclusivo del ámbito regional. Se evidenció una organización distinta a la revolución pingüina del año 2006, una unión entre los universitarios y secundarios bajo un mismo propósito.

Posteriormente a la etapa informativa de los medios comenzó un discurso más sensacionalista, más "crónica roja", es decir, se empezó a enfatizar en ambos medios los hechos ocurridos en las marchas estudiantiles, en las tomas de los establecimientos.

Se destacaba los incidentes en los enfrentamientos con Carabineros, el destrozo de propiedad privada como la sucursal del Banco Crédito Inversiones en el sector de Isla Teja, a pasos de la Universidad Austral de Chile. Las innumerables tomas del puente Pedro de Valdivia, cortes de calles en la cercanía del Liceo Técnico y del Liceo Industrial, quema de neumáticos realizados por encapuchados, etc.

Se invisibilizaron manifestaciones de carácter lúdicas y creativas, como el puerta a puerta realizado por estudiantes de las carreras de Pedagogía en Lengua Inglesa y de Derecho de la Universidad Austral de Chile, además el campeonato de fútbol calle realizado afuera de la Intendencia de Valdivia por estudiantes universitarios y secundarios.

Al mismo tiempo, las autoridades siempre fueron tratadas con conceptos como comprensivos, dialogantes, preocupados e interesados por el movimiento social. Pocas veces fueron interpelados en la noticia. 
Luego se dio paso a la última fase promovida por los dos medios de comunicación, el debilitamiento del movimiento y los destrozos en los establecimientos educacionales municipales. Cada vez que se suscitaba una nueva marcha estudiantil, éstos siempre utilizaban citas de autoridades de gobierno para estimar el número de participantes, y aunque ésta contara con dos mil personas, lo habitual era disminuir dicha cifra. Incluso se generaban discursos de división, de término del movimiento debido a que eran pocos los estudiantes secundarios y universitarios que seguían manteniendo vivo el movimiento estudiantil.

A su vez, podemos señalar que el tratamiento informativo realizado por El Diario Austral Región de Los Ríos fue más periodístico que el de El Naveghable, ya que en este último los corresponsales no tienen una formación periodística y se cometía el error de que la estructura de las noticias tenían un enfoque más de columna de opinión que de nota informativa.

Sin embargo, se puede mencionar que el tratamiento informativo del movimiento estudiantil en Valdivia estuvo enfocado en y a la criminalización de los estudiantes secundarios, ya que la mayoría de las noticias se referían a destrozos, barricadas, robos y gestores de bombas artesanales, hecho que estereotipa y genera un prejuicio en la comunidad, cambiando la visión de ésta sobre el conflicto.

En la investigación queda demostrado que los medios de comunicación ya han dejado y abandonado su rol netamente informativo y de responsabilidad social, han pasado a ser verdaderas empresas de producción y de publicidad. Según Gomis (1991), la comunicación de masas es una producción masiva de comunicación, la cual se rige por los mismos principios que las industrias: producción en serie, indiferenciada, a fin de reducir costes y aumentar beneficios. Hoy en día los medios generan un pensamiento acrítico, indiferenciado y único.

No obstante cabe señalar sobre este movimiento estudiantil que, a pesar de que los estudiantes no lograron obtener todo lo que solicitaban en su petitorio, fueron durante varios meses protagonistas claves en una de las materias más importantes de una nación, como es la educación, poniéndola como tema central en los noticiarios y entre la ciudadanía.

\section{Referencias bibliográficas}

BURGUET, Francesc (2004): La Trampa de los Periodistas. Barcelona, Edición 62.

BECHELLONI, Giovanni (1986): "Il potere nelle organizzazioni dei media". Problemi dell'informazione, no. 3.

FEIXA, Carles (2002): Movimientos juveniles: de la globalización a la antiglobalización. Barcelona, Ariel.

GOMIS, Lorenzo (1991): Teoría del Periodismo. Barcelona, Paidós Ibérica.

NEVEU, Erik (2000): Sociología de los movimientos sociales. Quito, Ediciones Abya Yala.

REGUILLO, Rossana (2003): Emergencia de Culturas Juveniles. Estrategias del desencanto. Bogotá, Norma. 
RODRIGO ALSINA, Miquel (1995): Los Modelos de la Comunicación. Madrid, Tecnos, $2^{\mathrm{a}}$ edición.

RODRIGO ALSINA, Miquel (2005): La Construcción de la Noticia. Barcelona, Paidós Ibérica.

ROMANO, Vicente (1991): La Formación de la mentalidad sumisa. Venezuela.

ROMANO, Vicente (2006): Comunicación, poder y democracia. Barcelona, Paidós Ibérica.

VALLEJO, Camila (2012): Podemos Cambiar el Mundo. Santiago de Chile, Editorial Ocean Sur.

VAN DIJK, Teun (1990): La noticia como discurso. Barcelona, Paidós.

VAN DIJK, Teun (1999): El análisis crítico del discurso. Barcelona, Paidós. 